\title{
Hybrid Force/Position Control applied to Automated Guiding Tasks at the Microscale
}

\author{
Kanty Rabenorosoa, Student Member, IEEE, Cédric Clévy, Member, IEEE, Philippe Lutz, Member, IEEE
}

\begin{abstract}
Force feedback control constitutes a promising solution for achieving fully automated micro-assembly. In this paper, we report the study of force feedback control used during guiding task. An experimental setup with two sensorized fingers is proposed. It enables to grasp a micropart of 2 $\mathbf{m m} \times \mathbf{5 0} \mu \mathrm{m} \times \mathbf{5 0} \mu \mathrm{m}$ in size and to estimate the lateral contact force. A simulator is developed to take into account the gripping forces evolution during a lateral perturbation. External hybrid force/position control is chosen for performing automated guiding tasks. Experimental results demonstrate the ability of the implemented controller and suitable design of microsystems.
\end{abstract}

\section{INTRODUCTION}

Development of complex microsystems is increasing in accordance with the need on MEMS/MOEMS. To fabricate them, the use of micro-assembly constitutes a promising approach [1], [2], [3] because it notably enables to integrate heterogeneous components issued from incompatible microfabrication processes. At the microscale or even the nanoscale, the use of force control gives helpful information for performing tasks. In fact, fully position based already shows very promising results but does not enable to take into account interaction forces [4] and surface forces like pull-off forces [5] that are greatly influent at the microscale. In addition, it is constrained by difficulty to integrate sensors with high resolution. Force control constitutes an approach with less constraints in workspace and recent developments of force sensor provide tools with the suitable force range (gripping forces, adhesion forces, pull-off forces) for the micromanipulation of microparts (a volume of manipulated microparts often smaller than $0.1 \mathrm{~mm}^{3}$ ). Interaction forces range is estimated from hundreds of nanoNewtons to a few milliNewtons.

In this aim, microrobotic operations like pushing, gripping, fine alignment, insertion directed by force control are currently investigated [4], [6], [7], [8] and results show the efficiency of tasks based on force control. Due to the recent developments of force sensor in the range of milliNewtons with submicrometric resolution, problems of signal to noise ratio, volume of the sensor, and integration of sensors in the micro-assembly station, there are no relevant works which proposed force feedback control for automated microassembly tasks. Considering the guiding task presented in our

This work was supported by the French region Franche-Comté under the MIAAMI project and OSEO-ANVAR.

Authors are with FEMTO-ST Inst., UMR CNRS 6174 - UFC /ENSMM / UTBM, Automatic Control and Micro-Mechatronic Systems depart.(AS2M department), Besancon, France rkanty, cclevy, plutz@femto-st.fr previous works [9], gripping forces enable to guarantee the stability of a manipulated micropart between both fingers of a microgripper during its guiding in a rail. This kind of task is useful for automated assembly of reconfigurable microoptical bench presented in [3] but can be applied to various other applications. The model of gripping forces perturbated by lateral contact force was presented. It was shown that the microgripper based on two sensorized fingers enables to measure the lateral contact force between the micropart and the rail. Additionally, the control strategy is established for ensuring that the micropart does not slide or rotate when the lateral contact happens during the guiding. Controlling the position along the rail and the contact force between a grasped micropart and a rail side can be achieved by hybrid force/position control. Our previous works have validated the estimation of lateral contact force, the control strategy, and the control scheme [9]. The objective of this paper is to pursue this promising approach and the control scheme is investigated in order to perform automated guiding task. Hybrid force/position control scheme has been previously studied at the macroscale through torque input control [10] and through inner position control [11], [12]. As far as goes our knowledge, no study has been done for ensuring automated tasks based on this scheme at the microscale. Micropositioning stages that are used on microrobotic manipulator are, in most cases, position controlled. Thus, an external hybrid force/position control based on inner position control is relevant. A complete automated guiding task using external hybrid force/position control is proposed in these works through a simulator development and experimental investigations.

Therefore, an experimental setup based on two sensorized fingers for achieving guiding task is presented. The model of gripping force evolution during lateral contact force is integrated on Matlab-Simulink model. The study of hybrid force/position control is proposed by simulation of the guiding task. Experimental investigations on hybrid control in the considered force range is proposed. This paper is organized as follows: in section II, the experimental setup to achieve guiding tasks is presented. Model integration and simulation results are detailed in section III. In section IV, experimental results are presented and comparison to simulation results is discussed.

\section{EXPERIMENTAL SETUP}

In this section, the experimental setup is proposed for performing guiding task (see Fig. 1). It is based on two force sensors from FemtoTools with measuring range \pm 2000 
$\mu \mathrm{N}$. Each finger consisting in force sensor works like a jaw of the microgripper and is mounted on $X_{1,2} Y_{1,2} Z_{1,2}$ linear stages. The control of the position of fingers along $Y$ enables to open/close the resulting microgripper. The manipulated micropart is $2000 \mu \mathrm{m} \times 50 \mu \mathrm{m} \times 50 \mu \mathrm{m}$ in size. The rail is



Fig. 1. Experimental setup proposed for achieving guiding tasks: a micropart is hold by two sensorized fingers. $X_{n} Y_{n} Z_{n}, X_{p}$ enable guiding motions of this micropart into a rail.

mounted on a microrobotic structure composed of $X_{s} Y_{s} Z_{s}$ coarse positioning, $Y_{p}$ large range but fine positioning, $X_{n} Y_{n} Z_{n}$ fine positioning, and $\theta$ rotation. The large range positioning stage is a $\mathrm{P} 625.1 \mathrm{CD}$ from Physik Instrumente with $500 \mu \mathrm{m}$ travel range and $1.4 \mathrm{~nm}$ in resolution. The fine positioning stage is a P-611.3 NanoCube with $100 \mu \mathrm{m}$ range and $1 \mathrm{~nm}$ in resolution. The rotation stage is a SmarAct SR-3610-S with $1.1 \mu^{o}$ of resolution. These three devices are sensorized and closed loop controlled. The rail width is adjustable from $0 \mu \mathrm{m}$ to $1 \mathrm{~mm}$ enabling to set up the axial play between the guided micropart and the rail.

Considering the micropart manipulated with this microgripper, initial gripping forces applied by each finger are named preload and noted $F_{y 10}, F_{y 20}$. They enable to hold the micropart between fingers of the microgripper. The displacement along $X$ enables to position the micropart to the desired position into the rail. When the contact between the rail and the micropart appears, the rail position along $Y$ has to be corrected for canceling the force generated by the contact to preserve the stability and position of the micropart hold by the microgripper. In the following this force is named lateral contact force. It has three components: $F_{x}, F_{y}, F_{z}$ and we consider that $F_{x}$ and $F_{z}$ are smaller than $F_{y}$. In addition, coupling measurements are negligible, thus, $F_{y}$ can be estimated through static force equilibrium along $Y$ giving $F_{y}=F_{y 2}-F_{y 1}$. In the following, the microgripper is fixed and the center of the microgripper is defined by a coordinate frame $X_{\text {micropart }} Y_{\text {micropart }} Z_{\text {micropart }}$. The guiding task is performed by actuating $X_{n}$ to move forward and by moving $Y_{n}$ for correcting when the contact happens (see Fig. 2). $Y_{p}$ is used for creating a perturbation for simulating misalignment of the rail and the guiding axis, nonlinearity of the move forward stage, and the microfabrication tolerance. $X_{\text {rail }} Y_{\text {rail }} Z_{\text {rail }}$ is the coordinate frame of the rail.

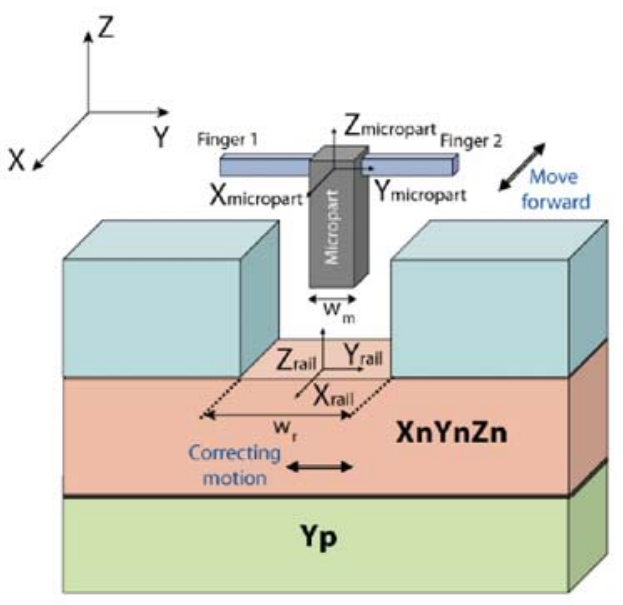

Fig. 2. Guiding task based on two sensorized fingers with the rail width $w_{r}$ and the micropart width $w_{m}$

\section{Simulation InVESTigation}

In this section, the model of gripping forces evolution when the contact appears (see Fig. 3) is integrated in a Matlab-Simulink model. A hybrid force/position control is proposed and simulator results are presented. These simulation results constitute reference data which will be compared to experimental investigations. Indeed the simulator is based on a reliable and precise model. The simulator facilitates the investigation of parameters influence and reduces the measurement procedures which are difficult at the microscale.
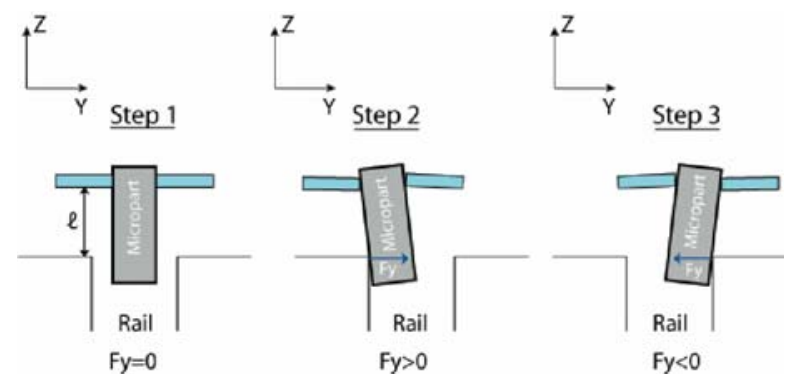

Fig. 3. Contact between the rail and the micropart during the guiding task: (Step 1) micropart does not touch the rail, (Step 2) left side contact, (Step 3) right side contact.

\section{A. Model integration}

1) Static evolution of gripping forces during the lateral contact: When a contact between the manipulated micropart and the rail appears, gripping forces change according to the contact force. The micropart displacement depends on the contact type between the probe and the micropart. Considering the planar micropart and the plan surface of the microgripper, it was shown in [9] that gripping forces evolution depend on several parameters: microgripper compliance $K_{x}, K_{y}, K_{z}$, microgripper fingers thickness $e_{p}$, distance of contact $\ell$, preload force... Gripping forces evolution for static case is integrated in a Matlab-Simulink model and takes into 
account the rail width and the micropart width. Results are shown in Fig. 4 with $20 \mu \mathrm{m}$ rail width, $10 \mu \mathrm{m}$ micropart width and $200 \mu \mathrm{N}$ preload. In this simulator based on Matlab-Simulink model, a white noise has been added to signals to make the study closer to the reality (noise has to be taken into account from the beginning of studies at the microscale).

When the perturbation is introduced, the positive contact

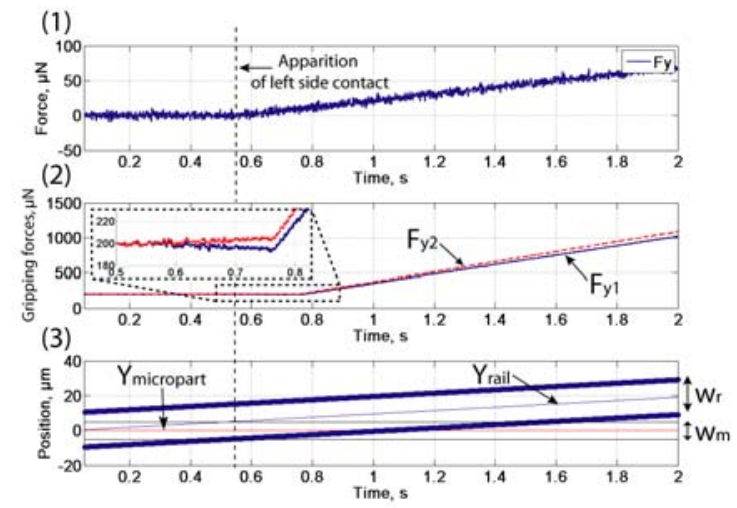

Fig. 4. Simulation of the system response in quasi-static :(1) Lateral contact force estimated by $F_{y}=F_{y 2}-F_{y 1}$, (2) gripping forces, and (3) micropart and rail position integrated to the simulator based on MatlabSimulink model.

force appears at $t=0.55 \mathrm{~s}$ and the situation corresponds to Step 3 in Fig. 3.

2) Dynamic behavior: In accordance of the microrobotic structure, the dynamic behavior is investigated and integrated to the simulator. Firstly, the dynamic response of the Nanocube (P-611.3) along $X$ and $Y$ is identified using OE (Output Error) model. Transfert functions are obtained and integrated to the simulator (1).

$$
N_{Y}(s)=N_{X}(s)=\frac{0.005 s^{2}+21.62 s+2212}{s^{2}+66.21 s+2212}
$$

Secondly, the dynamic behavior of the large positioning range $\mathrm{P} 625.1 \mathrm{CD}$ is also identified and his resonant frequency with the structure is determined at $35 \mathrm{~Hz}$ (2). The simulator result integrating these dynamic models is shown in Fig. 5 with $20 \mu \mathrm{m}$ rail width, $10 \mu \mathrm{m}$ micropart width, and $200 \mu \mathrm{N}$ preload.

$$
H_{P 625.1 C D}(s)=\frac{57.07 s+3383}{s^{2}+233.3 s+3383}
$$

\section{B. Hybrid force/position control for achieving guiding task}

The guiding task is automated by using an external hybrid force/position control. In this aim, the proposed block diagram (Fig. 6) enables to control the position in $X$ (move forward) and to remove the contact in $Y$. Indeed, $X_{d}=$ $[X, Y, Z]$ is the input position of the 3 DOF robot, $F_{d}$ is the input contact force $\left(F_{d}=0\right.$ in our case). The matrix of
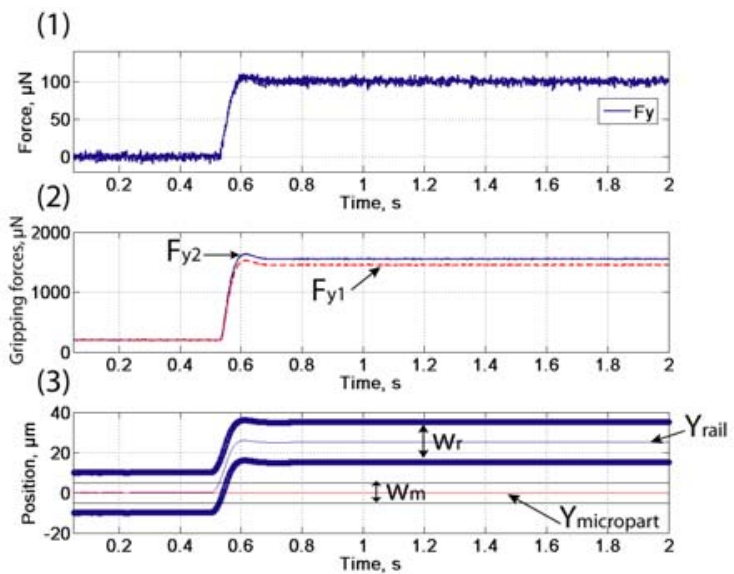

Fig. 5. Simulation of the system response in dynamic $\left(Y_{n}\right)$ : (1) lateral contact force estimation $\left(F_{y}=F_{y 2}-F_{y 1}\right)$, (2) gripping forces evolution $\left(F_{y 1}, F_{y 2}\right)$, (3) micropart position compared to the rail position along $Y$.

selection $\mathbf{S}$ enables the position control along $X$ and $Z$ :

$$
\left[\begin{array}{lll}
1 & 0 & 0 \\
0 & 0 & 0 \\
0 & 0 & 1
\end{array}\right]
$$

For avoiding the sliding or rotation of micropart during the

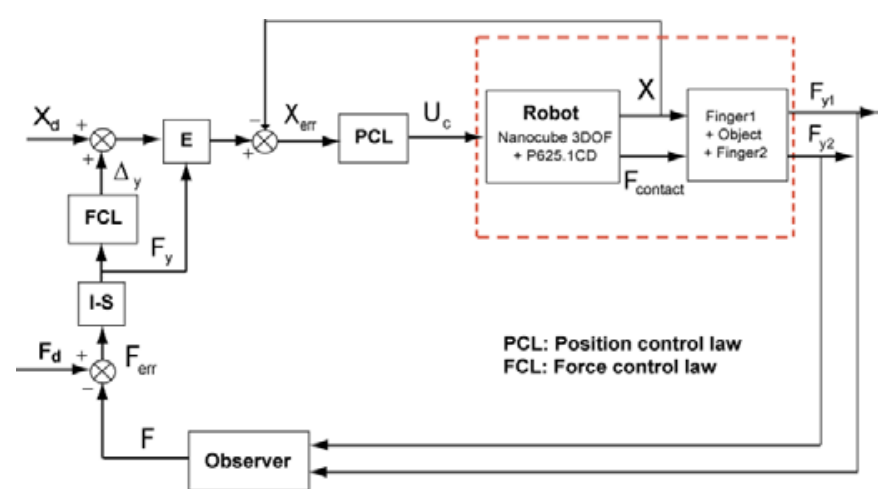

Fig. 6. Block diagram of the external hybrid force/position control during the guiding task.

contact, it is required to stop the motion along $X$ and to reduce the contact force under the limit shown in [9] (about $30 \mu \mathrm{N})$. The block $\mathbf{E}$ is the "enable control" which stops the motion along $X$ if the lateral contact force is bigger than this fixed limit. The details of $\mathbf{E}$ are shown in Fig. 7. Position



Fig. 7. Detailed of enable control. 
Control Laws (PLC) are Proportional Integral controllers. Investigations are focused on the Force Control law (FCL).

\section{Simulator results}

The use of Incremental Control is proposed for ensuring the control of the contact force. The study is done for different kind of perturbations. The complete system is not considered to be linear time invariant (LTI) due to the play between the micropart and the rail. Thus, conventional studies based on LTI theories are not relevant.

1) Incremental control: In the robotic field, the use of this controller enables easy and fast set up of parameters and reduces risks of breaking microparts or manipulator. It is composed of a dead zone for rejecting the sensor noise measurement $(15 \mu \mathrm{N})$, the sign operator indicates the direction of the increment, and the memory operation enables the relative positioning. Details are given in Fig. 8.

This controller enables to set the velocity of the correction

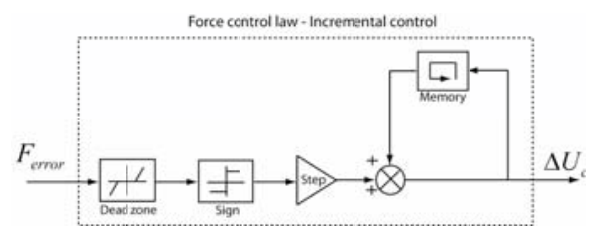

Fig. 8. Block diagram of incremental controller.

$V_{\text {corr }}$ in accordance with the sampling frequency $F_{\text {sampling }}$ and the increment $S t e p_{i n c r}$. It can be calculated by $V_{\text {corr }}=$ $F_{\text {sampling. Step }}$ incr.

2) Dynamic behavior: The system response to dynamic perturbations is investigated. The perturbation is generated by introducing a step of $20 \mu \mathrm{m}$ from $Y_{p}$ and the system reaction is observed in Fig. 9. The velocity of the correction is fixed at $100 \mu \mathrm{m} / \mathrm{s}$ ( $F_{\text {sampling }}=1000 \mathrm{~Hz}$ and Step $_{\text {incr }}=0.1$ $\mu \mathrm{m})$. The incremental control stability is also shown.

The increase of velocity correction induces the reduction of time for canceling the perturbation. This time is limited by the stage dynamic and the smallest time is about $35 \mathrm{~ms}$. Step incr $_{\text {has }}$ to be smaller than the play between the rail and the micropart for avoiding a contact apparition on the opposite side of the rail and the start of instabilities.

The effectiveness of "enable control" is observed in Fig. 9(3) -(4), the move forward along $X$ is stopped when the contact force is bigger than the fixed limit $(15 \mu \mathrm{N})$.

3) Ramp tracking: We have applied a ramp for simulating an alignment default between the rail and the move forward axis. The result is shown in Fig. 10 with $10 \mu \mathrm{m} / \mathrm{s}$ ramp slope. It is observed that $Y_{p}$ introduces the ramp perturbation and $Y_{\text {rail }}$ is maintained at certain distance of $Y_{\text {micropart }}$ for ensuring the contact force limit generated by a dead zone $(15 \mu \mathrm{N})$. The increase of gripping forces compared to the preload is estimated about $15 \%$ guaranteeing the stability of the micropart between microgripper fingers.
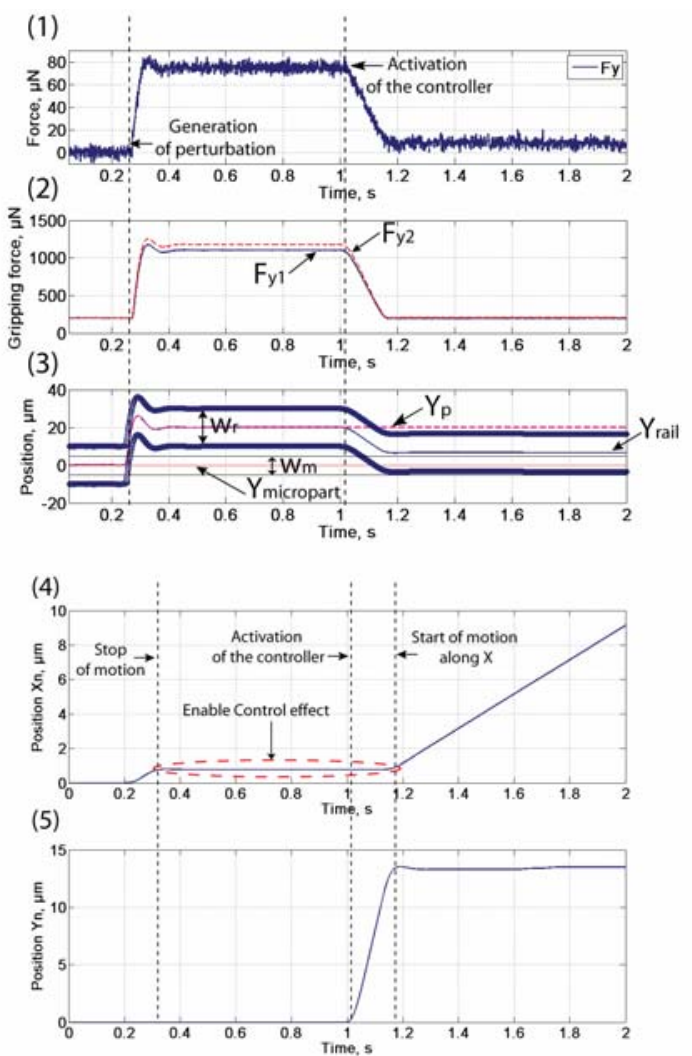

Fig. 9. Simulation of a guiding task: (1) lateral contact force estimation, (2) gripping forces evolution for left side contact, (3) micropart position compared to the rail position along $Y$, (4) move forward displacement with $10 \mu \mathrm{m} / \mathrm{s}$ velocity, (5) $Y_{n}$ displacement during the correction.

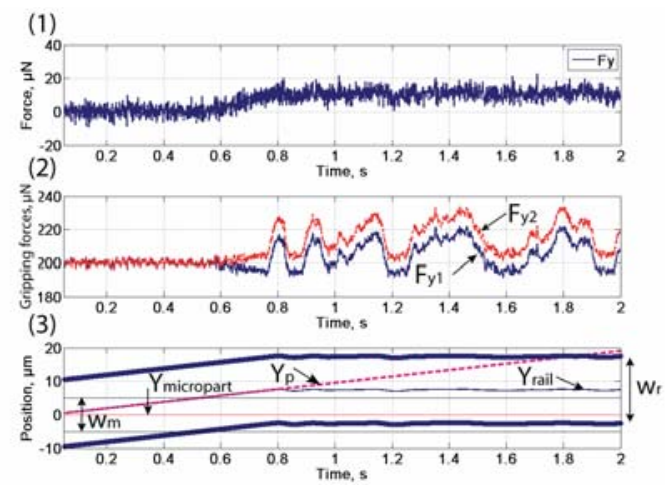

Fig. 10. Simulation of a guiding task: (1)lateral contact force estimation, (2) gripping forces evolution for left side contact, (3) micropart position compared to the rail position along $Y$.

\section{EXPERIMENTAL RESULTS}

In this section, experimental investigations are performed when the micropart is grasped with a defined preload. Observed behaviors during simulation will be checked and controller performances (enable control, correction velocity, increment step, sampling frequency) have to be validated. Firstly, the rail width is measured. Thus, the incremental control is used like a force control law in the external hybrid 
force/position control and perturbation effects are presented.

\section{A. Rail width measurement}

For measuring the rail width, a displacement of the substrate is generated with a fixed velocity and the contact force evolution is observed. The rail width corresponds to the micropart width added with the distance of two contact sides (see Fig. 11). The result of the rail width measurement is shown in Fig. 12. This result is used for illustrating the rail position and the micropart position in the following.

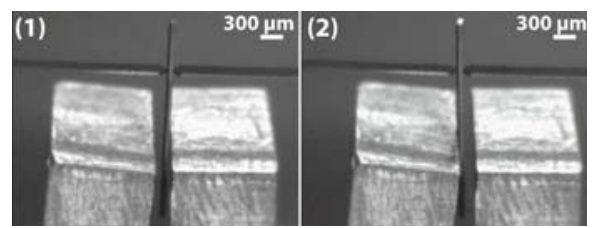

Fig. 11. Setup measurement of the rail width: (1) right side contact, (2) left side contact.

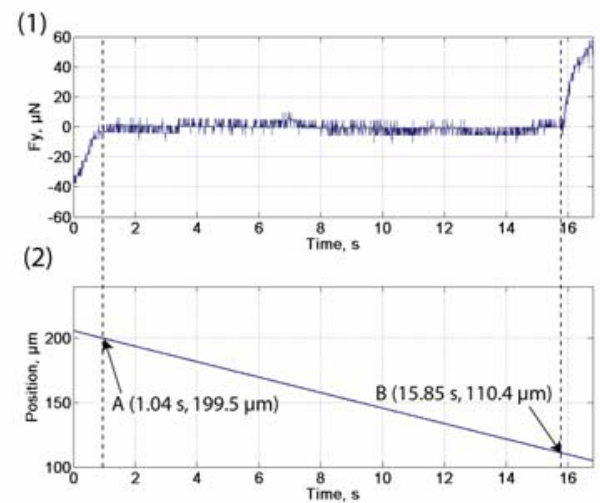

Fig. 12. Experimental measurement of rail width. Contacts happen at point $\mathrm{A}$ and $\mathrm{B}$, the micropart width is $50 \mu \mathrm{m}$, thus the rail width is $139 \pm 2 \mu \mathrm{m}$. (1) lateral contact force estimation $F_{y}=F_{y 2}-F_{y 2}$, (2) displacement along $Y$.

\section{B. Incremental Control}

The objective is to apply this controller on the force control law. The proposed incremental control is implemented on a 1103 Dspace board with a sampling frequency $F_{\text {sampling }}$ $=100 \mathrm{~Hz}$. The perturbation is generated by actuating $Y_{p}$ and

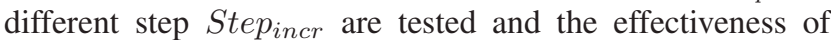
the control based on contact force estimation is validated. Results show that the time for canceling the perturbation is about $900 \mathrm{~ms}\left(\right.$ Step $\left._{\text {incr }}=0.275 \mu \mathrm{m}, F_{\text {sampling }}=100 \mathrm{~Hz}\right)$. The displacement of the rail along $X$ with a velocity of 1.90 $\mu \mathrm{m} / \mathrm{s}$ and $0.55 \mu \mathrm{m} / \mathrm{s}$ is performed.

The increase of velocity correction induces the reduction of this time and it is measured that it can reach $40 \mathrm{~ms}$. The obtained value is in the same order of the simulation results (35 ms). The effect of the enable control is checked with the force limit fixed at $15 \mu \mathrm{N}$. System behaviors observed experimentally correspond to the simulation results.

\section{Dynamic perturbation at each side of the rail}

The robustness of the guiding task control is tested by introducing a dynamic perturbation during the task. Left side contact and right side contact are generated successively during the move forward motion. The controller is already on at the beginning of the task. The fixed limit is here at 15 $\mu$ N. Results are shown in Fig. 13.
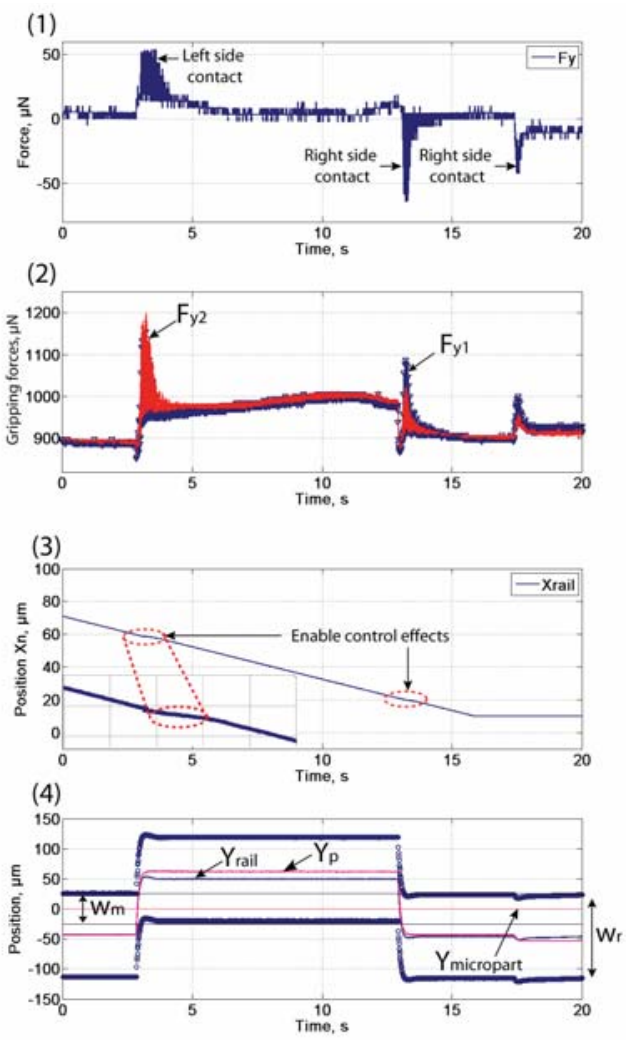

Fig. 13. Experimentation of a guiding task: (1) lateral contact force estimation $\left(F_{y}=F_{y 2}-F_{y 2}\right)$, (2) gripping forces evolution for left side and right side contact, (3) move forward displacement with $4 \mu \mathrm{m} / \mathrm{s}$ velocity, (4) micropart position compared to the rail position along $Y$ when left side and right side contact happen.

\section{Ramp tracking}

For simulating an alignment default between the rail and the move forward axis, we introduce a ramp by moving $Y_{p}$. During this phase, the controller is always on and can work directly. Results are shown in Fig. 14.

Considering the perturbation displacement and the the move forward displacement, an equivalent angle $\alpha$ of misalignment is estimated to $26.4^{\circ}$ by $\alpha=\tan ^{-1}\left(\Delta_{Y p} / \Delta_{X n}\right)$. It is observed that when the contact occurs, the estimated force gradually increases up to the fixed limit. The controller begins the correction for maintaining this force under the authorized limit. The micropart position is slightly out of the rail during this phase due to the offset contact force induced by the dead zone. If the contact appears in the other side, this offset contact force is about $-15 \mu \mathrm{N}$. We can also observe that during the guiding task, gripping forces are maintained 
(1)

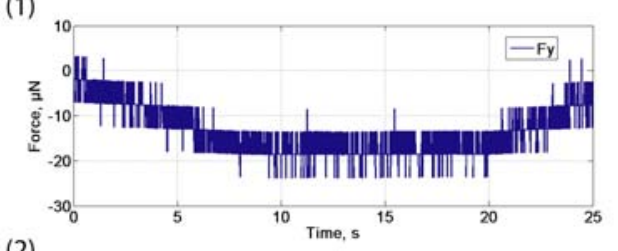

(2)

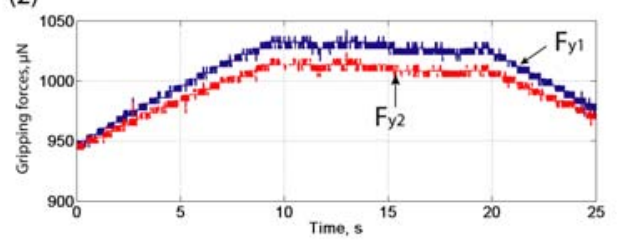

(3)

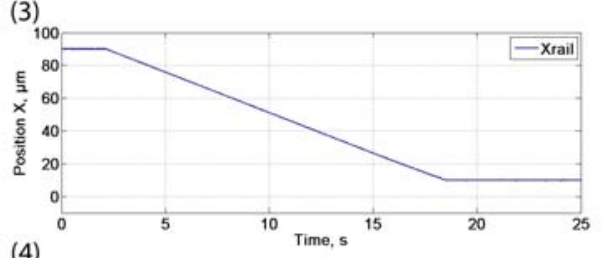

(4)

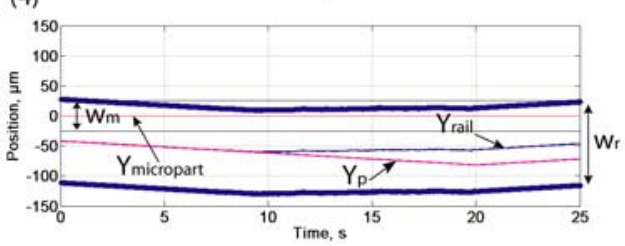

Fig. 14. Experimentation of a guiding task: (1) Lateral contact force estimation (2) gripping forces during the apparition of a ramp with $2 \mu \mathrm{m} / \mathrm{s}$ slope, (3) move forward displacement with $5 \mu \mathrm{m} / \mathrm{s}$ velocity, (4) micropart position compared to the rail position along $Y$.

in the tolerable range which enables to avoid the risk of breaking microparts, guarantee the stability of micropart between microgripper fingers. The increase compared to the preload is estimated to $7.9 \%$ for $15 \mu \mathrm{N}$ offset contact force. The position target along $X$ is reached without micropart sliding thus the task is achieved.

\section{CONCLUSION}

A study of force feedback based guiding task is proposed in this paper. The lateral contact force is estimated by using two sensorized fingers and is used in an external hybrid force/position control. A simulator development based on Matlab-Simulink model is investigated and the use of this simulator enables to set parameters of the controller. It prevents for breaking a part of the system if the control is unstable. The effectiveness of the incremental controller is validated and it was shown that the time for canceling a step perturbation can reach the corrector stage dynamic $(\approx$ $35 \mathrm{~ms}$ ). This controller depends on sampling frequency and step increment: the magnitude of this step has to be smaller than the play for ensuring the stability. An experimental setup is developed to perform the guiding task. A micropart of 2 $\mathrm{mm} \times 50 \mu \mathrm{m} \times 50 \mu \mathrm{m}$ in size is grasped, the incremental controller is validated by rejecting perturbations at each side of the rail. A ramp tracking with $2 \mu \mathrm{m} / \mathrm{s}$ slope which corresponds to a misalignment (equivalent angle about 26.4 ${ }^{\circ}$ ) between the rail and the guiding axis is performed. The slight increase of gripping forces during the task authorizes to perform it with fragile microparts and enables to ensure the stability of the micropart during the guiding (and therefore to ensure the relative positioning between the micropart and the microgripper frame). This whole study shows that the use of hybrid force/position control for achieving automated micro-assembly tasks constitutes an encouraging strategy. The developed simulator constitutes an interesting tool for investigating control domain and will be upgraded by introducing pull-off forces. The estimated contact force is done for 1 DOF and additional force information will be studied for performing complex automated assembly. Results obtained in this work can be extended to others tasks like insertion and micropart position control during joining indeed internal constraints of the glue generates undesired displacements.

\section{ACKNOWLEDGMENTS}

These works have partially been supported by the FrancheComté région under the MIAAMI project, and by the French National Research Agency (ANR) under the Smart Surface contract ANR-06-ROBO-009 . Authors would like to thank David Guibert for his technical support.

\section{REFERENCES}

[1] N. Dechev, W. Cleghorn, and J. Mills, "Microassembly of 3-d microstructures using a compliant, passive microgripper," J. of Microelec. Syst., vol. 13, pp. 176 - 189, 2004.

[2] A. Das, P. Zhang, W. H. Lee, H. Stephanou, and D. Popa, " $\mu^{3}$ : Multiscale, deterministic micro-nano assembly system for construction of on-wafer microrobots," IEEE International Conference on Robotics and Automation, pp. 461-466, 2007.

[3] S. Bargiel, K. Rabenorosoa, C. Clévy, C. Gorecki, and P. Lutz, "Towards the high accuracy assembly of hybrid moems components on a reconfigurable silicon free-space micro-optical bench," Journal of Micromechanics and Microengineering (JMM), In Press.

[4] Z. Lu, P. C. Y. Chen, A. Ganapathy, G. Zhao, J. Nam, G. Yang, E. Burdet, C. Teo, Q. Meng, and W. Lin, "A force-feedback control system for micro-assembly," Journal of Micromech. Microeng, vol. 16, pp. 1861-1868, 2006.

[5] K. Rabenorosoa, C. Clévy, P. Lutz, M. Gauthier, and P. Rougeot, "Measurement of pull-off force for planar contact at the microscale," Micro Nano Letters, vol. 4, pp. 148 -154, 2009.

[6] S. Khan and A. Sabanovic, "Hybrid vision/force feedback control for pushing micro-objects," in IEEE/RSJ IROS, 2009, pp. 577 - 582.

[7] W. H. Lee, B. H. Kang, Y. S. Oh, and H. Stephanou, "Micropeg manipulation with a compliant microgripper," in IEEE ICRA, 2003, pp. 3213-3218.

[8] G. Yang, J. A. Gaines, and B. J. Nelson, "A flexible experimental workcell for efficient and reliable wafer-level 3d microassembly," in IEEE ICRA, 2001, pp. 133-138.

[9] K. Rabenorosoa, C. Clévy, and P. Lutz, "Active force control for robotic micro-assembly: Application to guiding tasks," in IEEE ICRA, May 2010, pp. 2137-2142.

[10] M. Raibert and J. Craig, "Hybrid position/force control of manipulators," Trans. of ASME, J. of Dyn. Sys., Meas. and Cont., vol. 102, 1981.

[11] R. Volpe and P. Khosla, "A theoretical and experimental investigation of explicit force control strategies for manipulators," IEEE Trans. on Automatic Control, vol. 38, pp. 634-1650, 1993.

[12] V. Perdereau and M. Drouin, "A new scheme for hybrid force-position control," Lecture Notes in Control and Information Sciences, vol. 187, pp. 150-159, 1993. 